\title{
A Well-Balanced Numerical Model for the Simulation of Long Waves over Complex Domains
}

\author{
Gangfeng Wu1, Zhiguo He1,2*, Guohua Liu1 \\ ${ }^{1}$ Institute of Hydraulic Structures and Water Environment, Zhejiang University, Hangzhou, China \\ ${ }^{2}$ Ocean College, Zhejiang University, Hangzhou, China \\ Email: hezhiguo@zju.edu.cn, zjdxwgf@gmail.com
}

Received March 2014

\begin{abstract}
This paper presents a well-balanced two-dimensional (2D) finite volume model to simulate the propagation, runup and rundown of long wave. Non-staggered grid is adopted to discretize the governing equation and the intercell flux is computed using a central upwind scheme, which is a Riemann-problem-solver-free method for hyperbolic conservation laws. The nonnegative reconstruction method for water depth is implemented in the present model to treat the appearance of wet/dry fronts, and the friction term is solved by a semi-implicit scheme to ensure the stability of the model. The Euler method is applied to update flow variable to the new time level. The model is verified against two experimental cases and good agreements are observed between numerical results and observed data.
\end{abstract}

\section{Keywords}

Long Wave, Central Upwind Scheme, Well-Balanced, Wetting and Drying

\section{Introduction}

Long waves, such as storm surges, tides, or tsunamis, will cause huge casualties and considerable property damage. So it is important to develop an accurate and robust numerical model to predict and understand the propagation and runup of long wave. The nonlinear shallow water (NLSW) equations are widely employed to model the physical process of long wave [1-5]. Although the models based on NLSW omit dispersive effects, these models are able to provide the general characteristics of the wave runup process [4]. A good long wave model should have two major properties, which are crucial for the stability of numerical model [6]: 1) The model should be able to be well balanced; 2) The model should preserve the water depth to be nonnegative. Therefore, this paper presents a 2D well-balance shallow water model to simulate the propagation, runup and rundown of long wave. The model is able to preserve the "lake at rest" steady states and guarantee the positivity of the computed water depth.

"Corresponding author.

How to cite this paper: Wu, G.F., He, Z.G. and Liu, G.H. (2014) A Well-Balanced Numerical Model for the Simulation of Long Waves over Complex Domains. Journal of Applied Mathematics and Physics, 2, 418-424.

http://dx.doi.org/10.4236/jamp.2014.26050 


\section{Governing Equation}

The two-dimensional NLSW equations can be written as:

$$
\frac{\partial \boldsymbol{q}}{\partial t}+\frac{\partial \boldsymbol{f}}{\partial x}+\frac{\partial \boldsymbol{g}}{\partial y}=\boldsymbol{S}
$$

in which

$$
\boldsymbol{q}=\left[\begin{array}{c}
\eta \\
h u \\
h v
\end{array}\right], \boldsymbol{f}=\left[\begin{array}{c}
h u \\
h u^{2}+\frac{1}{2} g h^{2} \\
h u v
\end{array}\right], \boldsymbol{g}=\left[\begin{array}{c}
h v \\
h u v \\
h v^{2}+\frac{1}{2} g h^{2}
\end{array}\right], \quad \boldsymbol{S}=\left[\begin{array}{c}
0 \\
-g h \frac{\partial z_{b}}{\partial x}-S_{f x} \\
-g h \frac{\partial z_{b}}{\partial y}-S_{f y}
\end{array}\right]
$$

where, $\boldsymbol{q}$ represents the vector of conserved variables; $\boldsymbol{f}$ and $\boldsymbol{g}$ are the flux vectors associated with the conserved variables in the $x$ - and $y$-directions, respectively. $\boldsymbol{S}$ represents the vector of source terms. $t$ indicates time; $x$ and $y$ are Cartesian coordinates; $\eta$ is water surface elevation; $h$ is water depth; $z_{b}$ represents bed elevation over the datum where $\eta=h+z_{b} ; u$ and $v$ are depth-averaged velocity components in the $x$ - and $y$-directions, respectively; $g$ is the gravitational acceleration; $S_{f \mathrm{x}}$ and $S_{f y}$ are the friction source terms in the $x$ and $y$ directions, respectively. In this paper, the friction source terms are estimated by using the Manning's formula:

$$
S_{f x}=\frac{g u \sqrt{u^{2}+v^{2}} n^{2}}{h^{1 / 3}}, \quad S_{f y}=\frac{g v \sqrt{u^{2}+v^{2}} n^{2}}{h^{1 / 3}}
$$

where $n$ is the Manning coefficient.

\section{Numerical Method}

\subsection{Finite Volume Discretization for NLSW Equations}

The discretization of Equation (1) is based on the finite volume method. As shown in Figure 1, the model adopts the non-staggered structure grids, in which the conserved variables and bottom bed elevation are defined at the cell center and represent the average value of each cell. Integrating Equation (1) over the cell $i j$ and applying Green's theorem yields:

$$
\boldsymbol{q}_{i, j}^{k+1}=\boldsymbol{q}_{i, j}^{k}-\frac{\Delta t}{\Delta x}\left(\boldsymbol{f}_{i+1 / 2, j}-\boldsymbol{f}_{i-1 / 2, j}\right)-\frac{\Delta t}{\Delta y}\left(\boldsymbol{g}_{i, j+1 / 2}-\boldsymbol{g}_{i, j-1 / 2}\right)+\Delta t \boldsymbol{S}_{i, j}
$$

where the superscript $k$ is the time level, subscripts $i$ and $j$ are indices of the cell, $\Delta t, \Delta x$, and $\Delta y$ are the time step, and cell sizes in the $x$ and $y$ directions, respectively. $\boldsymbol{f}_{i+1 / 2, j}, \boldsymbol{f}_{i-1 / 2, j}, \boldsymbol{g}_{i, j-1 / 2}$ and $\boldsymbol{g}_{i, j+1 / 2}$ are the flux at the interface of the cell $i j . S_{i, j}$ represents the source terms evaluated at the cell center.

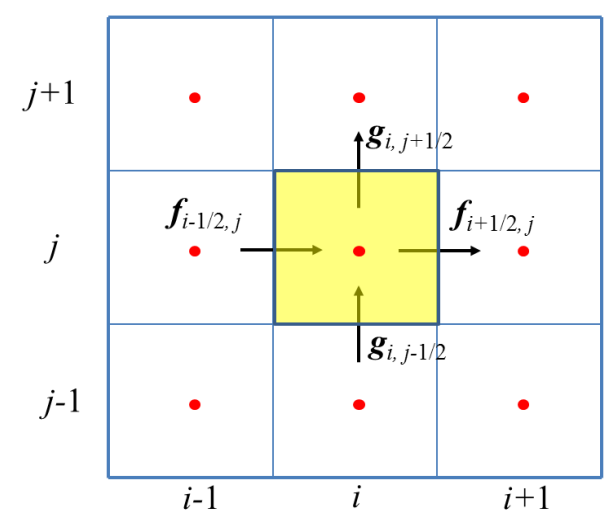

Figure 1. Sketch of control volume and structure grids. 


\subsection{Central Upwind Scheme}

In the present Godunov-type framework, the interface fluxes are calculated using a central upwind scheme, which requires the correct reconstruction of the Riemann states at the interface. The method of nonnegative reconstruction of water depth method proposed by Liang [7] is used to calculate the Riemann states. One can refer to [7] for more details. Then the interface flux can be calculated using central upwind scheme [8]:

$$
\begin{gathered}
\boldsymbol{f}_{i+1 / 2, j}=\frac{a_{i+1 / 2, j}^{+} \boldsymbol{f}\left(\boldsymbol{q}_{i+1 / 2, j}^{L},\left(z_{b}\right)_{i+1 / 2, j}\right)-a_{i+1 / 2, j}^{-} \boldsymbol{f}\left(\boldsymbol{q}_{i+1 / 2, j}^{R},\left(z_{b}\right)_{i+1 / 2, j}\right)}{a_{i+1 / 2, j}^{+}-a_{i+1 / 2, j}^{-}}+\frac{a_{i+1 / 2, j}^{+} a_{i+1 / 2, j}^{-}}{a_{i+1 / 2, j}^{+}-a_{i+1 / 2, j}^{-}}\left(\boldsymbol{q}_{i+1 / 2, j}^{R}-\boldsymbol{q}_{i+1 / 2, j}^{L}\right) \\
\mathbf{g}_{i, j+1 / 2}=\frac{b_{i, j+1 / 2}^{+} \mathbf{g}\left(\mathbf{q}_{i, j+1 / 2}^{L},\left(z_{b}\right)_{i, j+1 / 2}\right)-b_{i, j+1 / 2}^{-} \mathbf{g}\left(\mathbf{q}_{i, j+1 / 2}^{R},\left(z_{b}\right)_{i, j+1 / 2}\right)}{b_{i, j+1 / 2}^{+}-b_{i, j+1 / 2}^{-}}+\frac{b_{i, j+1 / 2}^{+} b_{i, j+1 / 2}^{-}}{b_{i, j+1 / 2}^{+}-b_{i, j+1 / 2}^{-}}\left(\mathbf{q}_{i, j+1 / 2}^{R}-\mathbf{q}_{i, j+1 / 2}^{L}\right)
\end{gathered}
$$

where $\boldsymbol{q}_{i+1 / 2, j}^{L}, \boldsymbol{q}_{i+1 / 2, j}^{R}$ are the reconstructed Riemann states on the left and right hand side of cell interface $(i+$ $1 / 2, j$ ), respectively. $\boldsymbol{q}_{i, j+1 / 2}^{L}, \boldsymbol{q}_{i, j+1 / 2}^{R}$ represent the reconstructed Riemann states on the left and right hand side of cell interface $(i, j+1 / 2)$, respectively. $a_{i+1 / 2, j}^{ \pm}, b_{i, j+1 / 2}^{ \pm}$are the one-sided local wave speeds in the $x$ and $y$ directions, respectively, and can be calculated as:

$$
\begin{aligned}
& a_{i+1 / 2, j}^{+}=\max \left\{u_{i+1 / 2, j}^{R}+\sqrt{g h_{i+1 / 2, j}^{R}}, u_{i+1 / 2, j}^{L}+\sqrt{g h_{i+1 / 2, j}^{L}}, 0\right\} \\
& a_{i+1 / 2, j}^{-}=\min \left\{u_{i+1 / 2, j}^{R}-\sqrt{g h_{i+1 / 2, j}^{R}}, u_{i+1 / 2, j}^{L}-\sqrt{g h_{i+1 / 2, j}^{L}}, 0\right\} \\
& b_{i, j+1 / 2}^{+}=\max \left\{v_{i, j+1 / 2}^{R}+\sqrt{g h_{i, j+1 / 2}^{R}}, v_{i, j+1 / 2}^{L}+\sqrt{g h_{i, j+1 / 2}^{L}}, 0\right\} \\
& b_{i, j+1 / 2}^{-}=\min \left\{v_{i, j+1 / 2}^{R}-\sqrt{g h_{i, j+1 / 2}^{R}}, v_{i, j+1 / 2}^{L}-\sqrt{g h_{i, j+1 / 2}^{L}}, 0\right\}
\end{aligned}
$$

where $u_{i+1 / 2, j}^{L}, u_{i+1 / 2, j}^{R}$ are the velocity in $x$ direction on the left and right hand side of cell interface $(i+1 / 2, j)$, respectively. $v_{i, j+1 / 2}^{L}, v_{i, j+1 / 2}^{R}$ are the velocity in $y$ direction on the left and right hand side of cell interface $(i, j+$ $1 / 2$ ), respectively.

\subsection{Discretization of the Source Terms}

The source term, as shown in Equation (2), can be split into the bed slope terms and friction terms. It is important to discretize the bed slope terms appropriately to ensure the scheme to be well balanced. Hence, the bed slope terms are approximated as follows:

$$
\begin{aligned}
& g h \frac{\partial z_{b}}{\partial x}=g \frac{\left(z_{b}\right)_{i+1 / 2, j}-\left(z_{b}\right)_{i-1 / 2, j}}{\Delta x} \cdot \frac{h_{i+1 / 2, j}^{L}+h_{i-1 / 2, j}^{R}}{2} \\
& g h \frac{\partial z_{b}}{\partial y}=g \frac{\left(z_{b}\right)_{i, j+1 / 2}-\left(z_{b}\right)_{i, j-1 / 2}}{\Delta y} \cdot \frac{h_{i, j+1 / 2}^{L}+h_{i, j-1 / 2}^{R}}{2}
\end{aligned}
$$

In general, a simple explicit discretization of friction term may cause numerical instability when the water depth is very small. To overcome this problem, the friction terms are discretized by using the semi-implicit treatment in this study. So the friction terms are discretized as follows:

$$
\begin{aligned}
& S_{f x}=\frac{g u \sqrt{u^{2}+v^{2}} n^{2}}{h^{1 / 3}}=\left(\frac{g \sqrt{u^{2}+v^{2}} n^{2}}{h^{4 / 3}}\right)^{k}(h u)^{k+1} \\
& S_{f y}=\frac{g v \sqrt{u^{2}+v^{2}} n^{2}}{h^{1 / 3}}=\left(\frac{g \sqrt{u^{2}+v^{2}} n^{2}}{h^{4 / 3}}\right)^{k}(h v)^{k+1}
\end{aligned}
$$

\subsection{Stability Criteria}

The current numerical scheme is explicit and its stability is governed by the Courant-Friedrichs-Lewy (CFL) criterion. It had been proved that the model could preserve the positivity of the water depth when the Courant 
number is less than 0.25 . One can refer [8] to for more details. Therefore, the CFL restriction for the current model is:

$$
N_{\mathrm{CFL}}=\max \left\{\frac{a \Delta t}{\Delta x}, \frac{b \Delta t}{\Delta y}\right\} \leq 0.25
$$

where $N_{\mathrm{CFL}}$ represents the Courant number, and $a$ and $b$ are given by:

$$
a=\max _{i, j}\left\{\max \left\{a_{i+1 / 2, j}^{+},-a_{i+1 / 2, j}^{-}\right\}\right\}, b=\max _{i, j}\left\{\max \left\{b_{i, j+1 / 2}^{+},-b_{i, j+1 / 2}^{-}\right\}\right\}
$$

\section{Numerical Results}

\subsection{Run-Up of a Solitary Wave on a Conical Island}

A series of experiments were performed by Briggs et al. [9] in a large scale basin at the US Army Engineer Waterways Experimental Station to study the run-up tsunami waves on a conical island. This case has been widely used to validate the wave runup model. The conical island, which had a base diameter of $7.2 \mathrm{~m}$, a top diameter of $2.2 \mathrm{~m}$, and a height of $0.625 \mathrm{~m}$, was located nearly the center of a $30 \mathrm{~m} \times 25 \mathrm{~m}$ basin. Planar solitary waves were produced by a directional wave-maker.

Since the reflected wave by the wall opposite to the wave makers is not investigated, a simplified $26.0 \mathrm{~m} \times$ $27.6 \mathrm{~m}$ computational domain is selected, as shown in Figure 2. Five water level gauges G1-G5 are located around the island to record the time histories of water surface elevation and their locations are given in Table 1. The island is submerged by the still flow with the depth of $0.32 \mathrm{~m}$ at the beginning. Following Nikolos et al. [10], the left boundary is set to be wave inflow boundary where the water level $\eta$ (herein water level is related to the still water) and velocity $u$ are given by

$$
\eta(t)=H \operatorname{sech}^{2}\left[\sqrt{\frac{3 H}{4 D}} C(t-T)\right]
$$

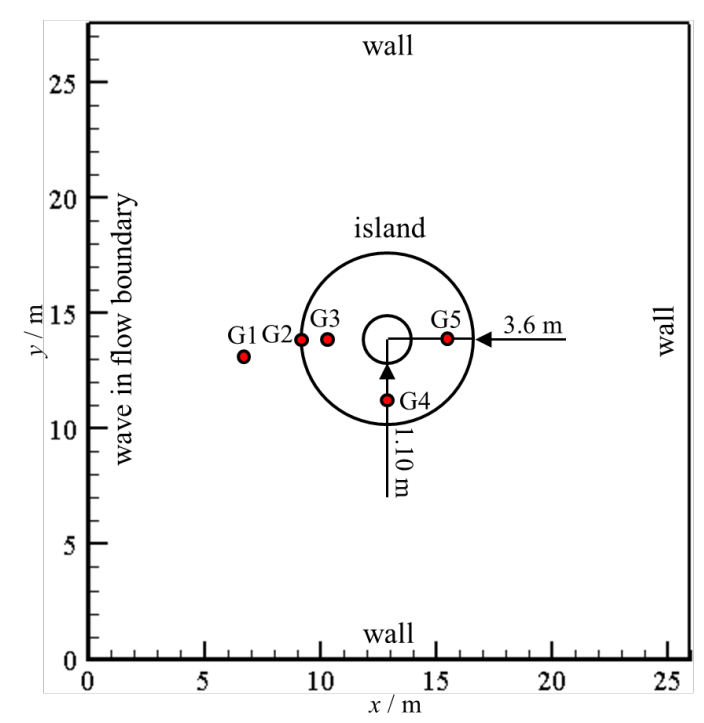

Figure 2. Computational domain, boundary conditions and the locations of the selected gauges.

Table 1. Locations of water level gauges.

\begin{tabular}{cccccc}
\hline Gauges & G1 & G2 & G3 & G4 & G5 \\
\hline$x / \mathrm{m}$ & 6.82 & 9.36 & 10.36 & 12.96 & 15.56 \\
$y / \mathrm{m}$ & 13.05 & 13.80 & 13.80 & 11.22 & 13.80 \\
\hline
\end{tabular}




$$
u(t)=\frac{C \eta}{D+\eta}, \quad v(t)=0
$$

where $H$ is the amplitude of the incident wave, $D$ is the still water depth, $T$ represent the time at which the wave crest enters the domain and $C=\sqrt{g(D+H)}$ is the wave celerity. In this study, a non-breaking incident wave is chosen with $H=0.032 \mathrm{~m}, D=0.32 \mathrm{~m}$ and $T=2.45 \mathrm{~s}$. The rest boundaries are set to be closed as shown in Figure 2. The computational domain is approximated by $260 \times 276$ grids with a uniform grid size of $0.1 \mathrm{~m}$. The simulation is carried out until 20s and the time step is determined by the CFL criteria. The computed and measured water levels at five gauges are shown in Figure 3. It can be found that the lead wave height and arrival time are well predicted by our model in most gauges. Some discrepancies between the simulated and measured water level may be attributed to the three-dimensionality of the wave in reality cannot be exactly captured by a depthaveraged 2D model. Overall the present model is capable to simulate the wave runup over complex topography with a satisfactory accuracy.

\subsection{Tsunami Runup onto a Complex 3D Beach}

This experiment is proposed and suggested as a benchmark test for numerical model in The Third International Workshop on Long Wave Run-up Models in 2004. The experiment was performed in a 1:400 scale wave tank, which approximated the coastline topography near Monai in Japan. As shown in Figure 4, the tank was $5.488 \mathrm{~m}$ long and $3.402 \mathrm{~m}$ wide. The incoming wave was generated by the movements of wave paddles and three gauges $\mathrm{Ch} 5, \mathrm{Ch} 7$, and $\mathrm{Ch} 9$ (see Figure 4) were setup to record the time history of water elevation.

For this case, the computational domain is approximated by $392 \times 243$ grids with a uniform grid size of 0.014 $\mathrm{m}$. At the beginning, the tank is filled with a still water of $0.135 \mathrm{~m}$ depth. The left boundary is wave inflow boundary, in which the time history of measured surface elevation was specified. The other three sides' boundaries are set to be closed. The total simulation time is $22.5 \mathrm{~s}$ and the manning coefficient is $n=0.0025$. The computed surface elevation at different time is plotted in Figure 4. Figure 5 shows the computed water elevation at three gauges compared with the observation data. In general, the movement of wave is well predicted by our model. Some discrepancies are found between numerical and experimental results due to the fact that the threedimensionality of the flow cannot be exactly captured by a depth-averaged 2D model. Nevertheless, the lead wave height and arrival times are predicted with a good accuracy at these gauges.

\section{Conclusion}

In this paper, a well-balanced numerical model is developed to simulate the long wave runup process. The central upwind scheme, which is a Riemann-problem-solver-free method, is used to calculate the flux at the interface. The model is able to preserve the "lake at rest" steady states and guarantee the positivity of the computed water depth when the Courant number is less than 0.25 . The model is validated against a solitary wave runup

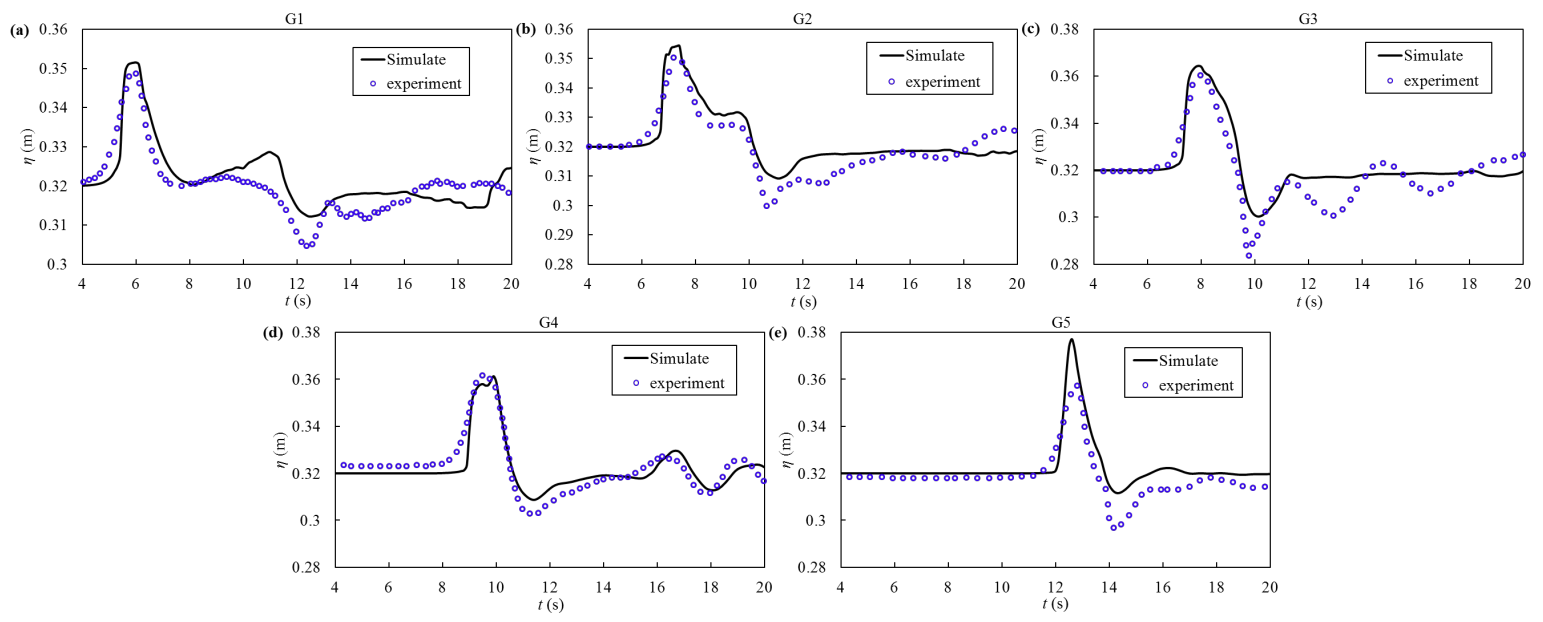

Figure 3. Time histories of water level at different gauges. 

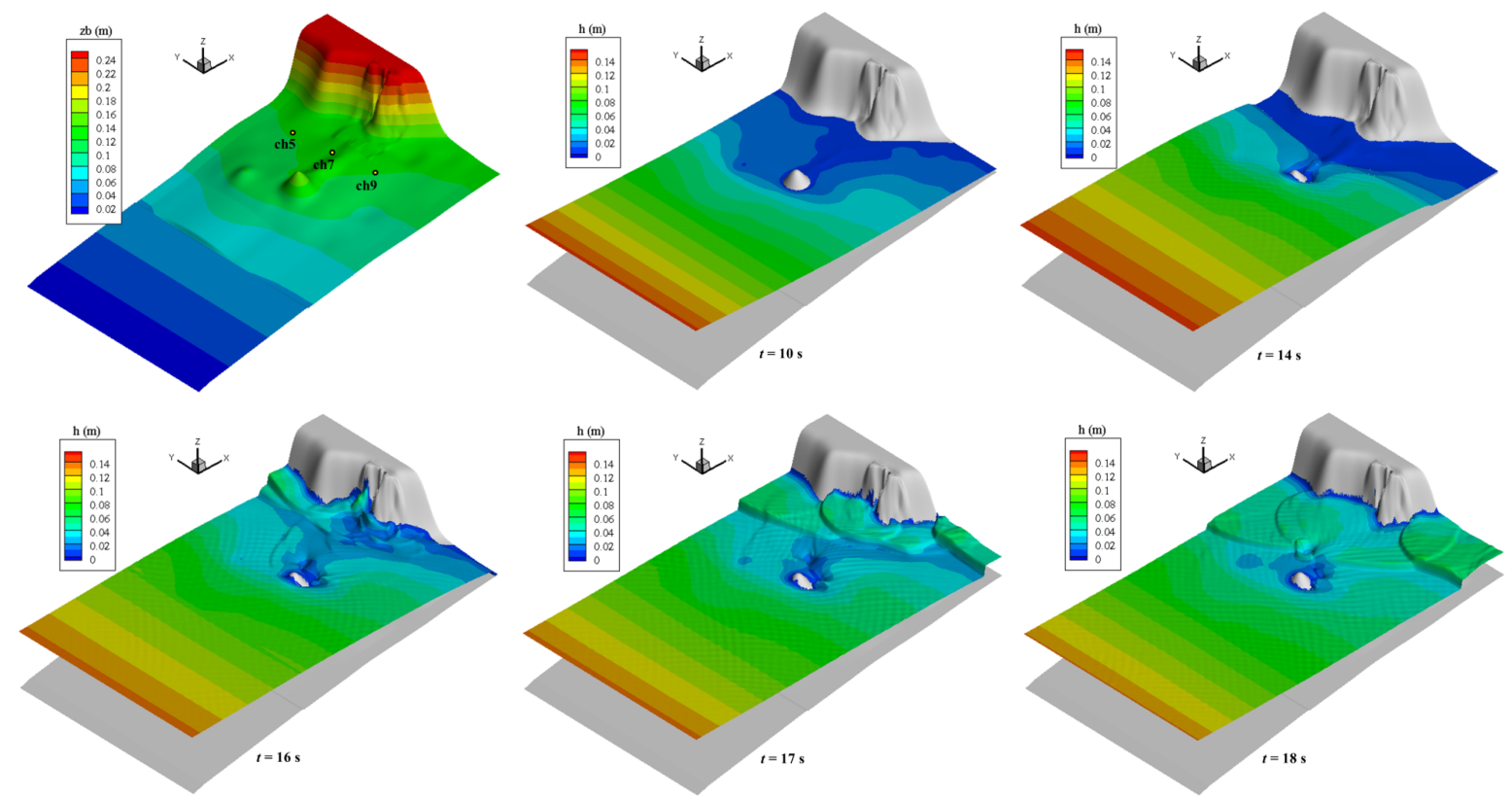

Figure 4. Bed elevation, the locations of gauges and the inundation map at different time.
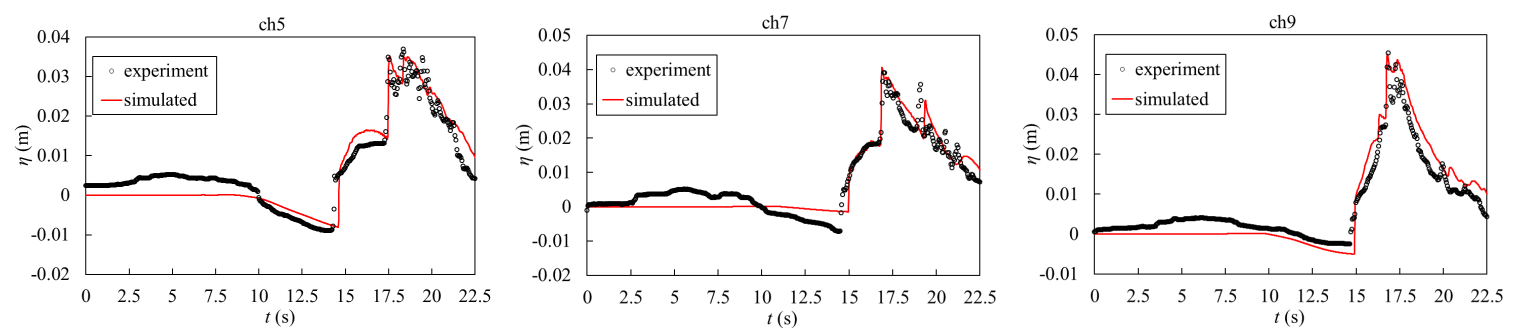

Figure 5. The computed water elevation at different gauges.

experiment and tsunami runup experiment. For both experiments, the simulated results agree very well with measurement data, which confirm the applicability of the present model for long wave runup applications.

\section{Acknowledgements}

This work is part of the research project sponsored by the National Basic Research Program of China (973 Program) (No 2013CB035900), Natural Science Foundation of China (51009120), Zhejiang Province Ocean and Fisheries Bureau (2010210), and Zhejiang University (2012HY012B).

\section{References}

[1] Dodd, N. (1998) Numerical Model of Wave Run-Up, Overtopping, and Regeneration. ASCE Journal of Waterway, Port, Coastal and Ocean Engineering, 124, 73-81. http://dx.doi.org/10.1061/(ASCE)0733-950X(1998)124:2(73)

[2] Hu, K., Mingham, C.G. and Causon, D.M. (2000) Numerical Simulation of Wave Overtopping of Coastal Structures Using the Non-linear Shallow Water Equations. Coastal Engineering, 41, 433-465. http://dx.doi.org/10.1016/S0378-3839(00)00040-5

[3] Hubbard, M.E. and Dodd, N. (2002) A 2D Numerical Model of Wave Run-up and Overtopping. Coastal Engineering, 47, 1-26. http://dx.doi.org/10.1016/S0378-3839(02)00094-7

[4] Delis, A.I., Kazolea, M. and Kampanis, N.A. (2008) A Robust High-Resolution Finite Volume Scheme for the Simulation of Long Waves over Complex Domains. International Journal for Numerical Methods in Fluids, 56, 419-452. http://dx.doi.org/10.1002/fld.1537

[5] Liang, Q., Wang, Y. and Archetti, R. (2010) A Well-Balanced Shallow Flow Solver for Coastal Simulations. International Journal of Offshore and Polar Engineering, 20, 41-47. 
[6] Bryson, S., Epshteyn, A., Kurganov, A. and Petrova, G. (2010) Well-Balanced Positivity Preserving Central-upwind Scheme on Triangular Grids for the Saint-Venant System. ESAIM: Mathematical Modelling and Numerical Analysis, 45, 423-446. http://dx.doi.org/10.1051/m2an/2010060

[7] Liang, Q. (2010) Flood Simulation Using a Well-Balanced Shallow Flow Model. ASCE, Journal of Hydraulic Engineering, 136, 669-675. http://dx.doi.org/10.1061/(ASCE)HY.1943-7900.0000219

[8] Kurganov, A. and Petrova, G. (2007) A Second-Order Well-Balanced Positivity Preserving Scheme for the Saint-Venant System. Communications in Mathematical Sciences, 5, 133-160. http://dx.doi.org/10.4310/CMS.2007.v5.n1.a6

[9] Briggs, M., Synolakis, C., Harkins, G. and Green, D. (1995) Laboratory Experiments of Tsunami Runup on a Circular Island. Pure and Applied Geophysics, 144, 569-593. http://dx.doi.org/10.1007/BF00874384

[10] Nikolos, I. and Delis, A. (2009) An Unstructured noDe-Centered finite Volume Scheme for Shallow Water Flows with Wet/Dry Fronts over Complex Topography. Computer Methods in Applied Mechanics and Engineering, 198, 37233750. http://dx.doi.org/10.1016/j.cma.2009.08.006 